\title{
Recurrent peritonitis with bowel Involvement mimicking bowel Ischemia
}

Keywords: autosomal, abdominal pain, gastrointestinal, manifestation, disease, edema, angioedema, deficiency, respiratory system, complications

Abbreviations: HAE, hereditary angioedema; C1-INH, c1esterase 1nhibitor; ED, emergency department; SAAG, serum ascites albumin gradient; FMF, familial mediterranean fever

\section{Introduction}

Hereditary angioedema (HAE) is a rare autosomal dominant disease characterized by recurrent episodes of angioedema that is caused by a deficiency (type 1) or dysfunction (type 2) of complement C1-esterase inhibitor (C1-INH). ${ }^{1}$ Respiratory system edema can result in serious complications, such as laryngeal edema, and hence, $30 \%$ of HAE patients die due to asphyxiation. ${ }^{2}$ On the other hand, HAE cases may also be present with abdominal pain. In fact, gastrointestinal involvement may be the only manifestation of the disease in one out of five cases. Gastrointestinal tract edema might manifest as severe acute onset abdominal pain or as chronic recurrent abdominal pain of moderate severity. ${ }^{3}$ We aimed to present a patient who had recurrent abdominal pains which was a difficult case to diagnose.

\section{Case report}

A 57year-old male presented to the Emergency Department (ED) with abdominal pain and nausea for 12 hours. He had been hospitalized several times for abdominal pain in many different centers. In the ED, he was found to have moderate tenderness in all quadrants with a mild leukocytosis. His amylase level was 342U/L (normal: 23-85U/L) and lypase level was 550U/L (normal: 0-160U/L). He was admitted to the gastroenterology clinic with an initial diagnosis of pancreatitis and started on intravenous hydration. Ultrasonography revealed a normalappearing pancreas and free fluid in Morison's pouch and his right lower quadrant. In the left upper and lower quadrants, the bowel wall appeared diffusely thickened and echogenic with a narrowed, hypoechoic lumen. A diagnostic paracenthesis was performed and his serum ascites albumin gradient (SAAG) was found to be lower than 1.1. Peritoneal fluid cytologic examination revealed reactive mesotelial cells and no sign of malignancy. CT scan of the abdomen following intravenous and oral administration of contrast medium revealed a regular wall thickening of the duodenum and proximal jejunum with thickened mucosal folds (Figure 1). A CT Angiography was performed with the initial diagnosis of bowel ischemia. However, mesenteric vessels were totally normal. Five days after his admission, his abdominal pain completely relieved and Ultrasonograpy showed that his intestinal walls had returned to normal and ascites had disappeared. He was discharged but referred again with same complaints four days later. He was found to have tenderness in all quadrants with a mild leukocytosis and the bowel wall appeared diffusely thickened on Ultrasonography just like his previous admission. An upper gastrointestinal endoscopy was performed. Duodenal and jejunal wall biopsies revealed mild inflammation. His colonoscopy was totally normal. He was discharged after his symptoms improved. On his third hospitalisation with same reason on our center,
Volume 2 Issue 3 - 2017

\author{
Enver Üçbilek,' Fehmi Ateș,' Orhan Sezgin,' \\ Engin Altıntaș,' Mahmut Bakır Koyuncu² \\ 'Department of Gastroenterology, Mersin University School of \\ Medicine, Turkey \\ ${ }^{2}$ Department of Internal Medicine, Mersin University School of \\ Medicine, Turkey
}

\begin{abstract}
Correspondence: Engin Altintas, Department of Gastroenterology, Mersin University School of Medicine, Mersin, Turkey, Email enginaltintas@gmail.com
\end{abstract}

Received: January 16, 2017 | Published: March 08, 2017

a diagnostic laparotomy was performed and no gross pathology other than edematous omentum was observed. Omental biopsy revealed congestion, mature adipose tissue with minimal inflammation. To exclude the diagnosis of Familial Mediterranean Fever (FMF) which is common in Turkey and is a cause of recurrent peritonitis, FMF mutation panel with common mutations was requested and found to be normal. He was discharged after his abdominal pain gradually decreased and his laboratory results normalized. 5days after discharge, he presented with the same clinical picture and he was suspected of having hereditary angioedema and a reduced C1-INH protein level of $4 \mathrm{mg} / \mathrm{dL}$ (Normal: $11-26 \mathrm{mg} / \mathrm{dL}$ ) was detected together with markedly lower C1-INH activity of $<10 \%$ (Normal: $70-130 \%$ ) and he was diagnosed with hereditary angioedema. He started on danazol. After Danazol treatment, he only had a mild episode over 3years of followup.

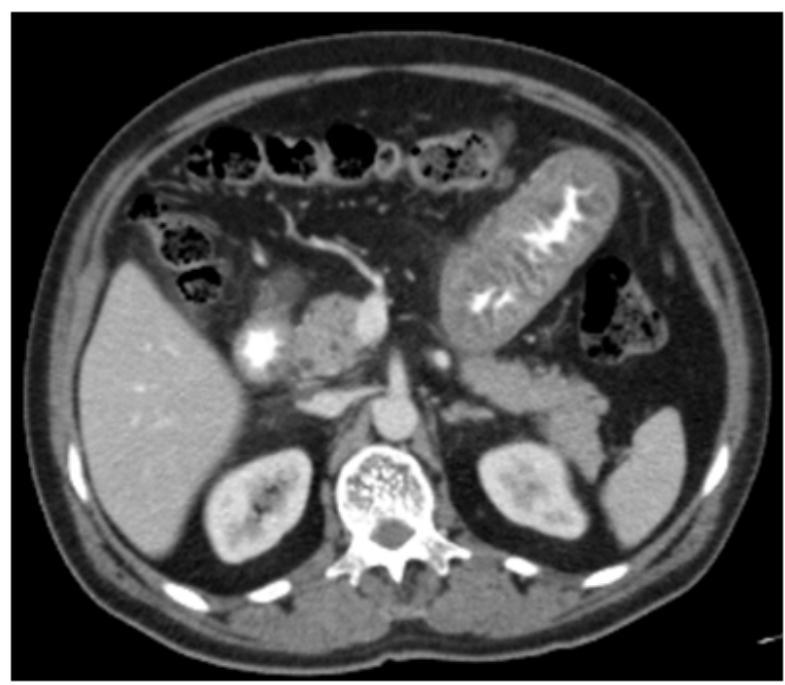

Figure 1 Regular wall thickening of the duodenum and proximal jejunum with thickened mucosal folds on CT scan. 


\section{Discussion}

Gastro-intestinal involvement is less frequent than angioedema of the face or the upper airways and may be the only manifestation of the disease in $21 \%$ of cases of hereditary angioneurotic edema. In the most cases of gastro-intestinal angioedema, the small bowel or duodenum is affected. The symptoms are often nonspecific and consist of recurrent abdominal pain, abdominal distension, nausea and vomiting. Sometimes, angioedema can mimic a (sub)acute abdomen. Therefore making the right diagnosis can prevent unnecessary invasive interventions.

In this case, there was no history of recurrent edema of the cutis or mucosa of the upper airways. The differential diagnosis of the presentation of the patient includes acute abdomen, bowel ischemia, FMF, Henoch-Schönlein vasculitis, intramural bleeding from trauma, anticoagulation therapy or hemophilia. CT angiography and colonoscopic examination showed no evidence of ischemia. FMF mutation panel was normal. He had no history of trauma or taking anticoagulation therapy. He had no family history or sign of hemophilia. After exclusion of all of the conditions which were on the list of differential diagnosis, the clinical picture with recurrent appearance and resolution of the symptoms led us to doubt HAE with isolated gastrointestinal involvement. The diagnosis was confirmed with measurement of serum levels of C1-esterase inhibitor and C1esterase inhibitor functional activity levels.

In the following three years, he only had one abdominal attack and $\mathrm{C} 1-\mathrm{INH}$ concentrate was necessary as his treatment for skin and oropharyngeal attacks. Although the relationship between HAE attacks and C1-INH concentrate injection is unclear, the observed decrease in the frequency of attacks might have been due to the frequent injections of $\mathrm{C} 1-\mathrm{INH}$ concentrate.

\section{Conclusion}

We encountered a case of HAE involving recurrent abdominal pain. Due to the fact that HAE is rare and HAE with isolated gastrointestinal involvement is even more rare we think that the most significant step in diagnosis is to suspect the disease. Peritoneal irritation signs and radiological evidence of increased bowel wall thickness in the absence of bowel ischemia should raise suspicion about this condition.

\section{Acknowledgements}

None.

\section{Conflict of interest}

Author declares that there is no conflict of interest.

\section{References}

1. Donaldson VH, Evans RR. A biochemical abnormality in hereditary angioneurotic edema: absence of serum inhibitor of $\mathrm{C} 1$ - esterase. $\mathrm{Am} \mathrm{J}$ Med. 1963;35:37-44.

2. Moore GP, Hurley WT, Pace SA. Hereditary angioedema. Ann Emerg Med. 1988;17:1082-1086.

3. Sim TC, Grant JA. Hereditary angioedema: its diagnostic and management perspectives. Am J Med. 1990;88(6):656-664.

4. Mai C, Claikens B, Van Wettere P. Intestinal angioneurotic edema. JBRBTR. 2014;97(3):162-163. 